\title{
Introduction to a Special Issue on Rural Health in the U.S.
}

\section{J. Matthew Fannin and Paul E. McNamara*}

Rural health is a subject area that has long been the domain of scholars in schools of public health and of those in the health care services research fields. Issues such as health care supply, patient demand, and access to in-patient and clinical services have been long-running research questions of scholars that focus on this area.

Regional scientists have made historical contributions to this area. For example, Gerald Doesken is notable for his application of input-output analysis to identify the economic contributions of hospitals and their employees to local rural economies in his extension programming activities. However, the regional science community has not made health care a focused area of long-term scholarship similar to subject areas such as migration, poverty, and economic growth. Yet, health care, and rural health care in particular, has spatial and institutional contexts that regional scientists can apply our theories and methods in order to provide important insights to proposed national health care policy and regional access and consumption questions.

To increase the regional science community's exposure to existing research in the area, a collection of sessions focusing on spatial and institutional factors affecting health care was organized at the 2008 meetings of the Southern Regional Science Association. The authors were invited to submit these papers for a special issue of The Review of Regional Studies. The papers in this special issue are the culmination of this effort.

The first two papers in the issue address whether rurality alone has differential effects on health outcomes and employer health care insurance decisions. In the first paper, Shields, Mushinski and Davis simultaneously measure the decision by an employer to offer health insurance and their expected health insurance costs. After controlling for other factors, they found that being a rural employer had no statistically significant effect on the likelihood of offering health insurance or on health insurance costs. However, factors that are more likely observed in rural areas, like lower county employment growth rates, lowered competition for employees in the labor market and reduced the likelihood that employers would offer health care insurance.

In the second paper, Asirvatham applies quantile regression techniques to evaluate the differential factors driving caloric intake, Body Mass Index, and Healthy Eating Index changes based on rural/urban differences, multi-state regional differences and demographic and environmental factors. The quantile approach helps to control for the difference between the interpretation (and policy options available) for individuals on the lower end of the distribution where those with low caloric intake face malnutrition challenges while those at the upper end of the distribution face challenges of obesity. His results indicate that rural residents have lower diet quality but no statistically different caloric intake relative to their urban counterparts.

\footnotetext{
* Fannin is Associate Professor of Agricultural Economics and Agribusiness at the Louisiana State University Agricultural Center. McNamara is Associate Professor of Consumer and Family Economics at the University of Illinois at UrbanaChampaign.

Contact author: J. Matthew Fannin, Department of Agricultural Economics and Agribusiness, 101 Agricultural Administration Building, LSU AgCenter, Baton Rouge, LA, 70803. Email: mfannin@agcenter.lsu.edu.

(C) Southern Regional Science Association 2010.

ISSN 1553-0892

SRSA, 1601 University Avenue, PO Box 6025, Morgantown, West Virginia 26506-6025, USA.
} 
Demographic factors such as lower educational attainment, more prevalent in rural areas, suggest challenges in interpreting nutritional labeling, resulting in low diet quality outcomes.

The next two papers use spatial proximity to identify health care supply and demand choices. In the third paper, Jintanakul and Otto address the question of whether rural residents choose their closest rural hospital option for health care services. They apply data on distance traveled from patient residence to the hospital where inpatient and outpatient services were provided. Their results show that rural residents are most likely to by-pass their nearest hospital for services if the resident has private insurance or if the condition treated is complex (e.g. heart attack). Rural residents are more likely to use their closest rural hospital if the procedure is performed on an out-patient basis.

In the fourth paper, Fannin and Barnes evaluated the choice of contractual arrangements between hospitals and physicians. They find factors such as the percent of Medicaid admissions statistically increasing the probability of non-metropolitan hospitals choosing market contractual arrangements with physicians as compared to their metropolitan counterparts. Further, by applying Geographically Weighted Regression, they find that much heterogeneity exists in the magnitude and signs of parameter estimates across space. In particular, they find that New England and Middle Atlantic hospitals are more likely to choose market arrangements with their physicians if other hospitals as far as 30 miles away also use market-like contractual arrangements with physicians, as compared to the South Midwest and Western regions of the country.

In the final paper, Wynveen conducts a case study regional analysis of Mississippi Delta counties to understand factors driving households to go outside of their own county for health care services. Phone interviews of households indicated availability of care and quality as the reasons most cited for seeking health care out-of-county. Yet, measurable heterogeneity existed in these factors between counties suggesting that "one size fits all” health policy solutions may be inappropriate for regions typically considered homogenous based on demographic and economic characteristics.

We hope that you enjoy the papers in this special issue. With recent comprehensive health reform occurring in the US, we believe there will be even greater demand for quality regional health care scholarship in the future. We hope that more regional scientists will consider expanding their research areas toward this important topic. 